\title{
Final Solution in Femoroacetabular Impingement - Threaded Cups in Hip Revision Surgery 2002-2012
}

\author{
Štěpán Magersky* \\ Department of Orthopedics, Charles University and Na Bulovce Hospital, Europe
}

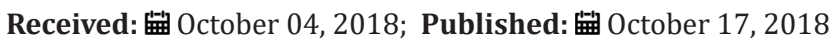

*Corresponding author: Štěpán Magersky, Department of Orthopedics, 1st Faculty of Medicine, Charles University and Na Bulovce Hospital, Budínova 2, Prague 8, 180 81, Czech Republic, Europe

\begin{abstract}
Purpose: Here, we report the complications of endoprosthesis with threaded cups according to our application in cases of complicated acetabulum (hip revisions and femoroacetabular impingement (FAI)).

Methods: A total of 504 patients was analyzed, including 189 men and 315 women. For re-implanted patients (n=49), the mean time to re-implantation was 6.3 years and the average age at re-implantation was 54.7 years. For censored patients ( $\mathrm{n}=455$ ), the mean time to censoring was 5.5 years and the average age of the implant was 55.7 years. Among the patients that were censored, 77 died and 378 patients did not experience an event necessitating re-implantation.

Results: For the total study population, the Kaplan-Meier estimate of 5-year survival is 0.94 and

of 10 -year survival is $0.85 .90 \%$ of the implants survived 7 years, $80 \%$ of the implants survived almost 12 years and $70 \%$ of implants survived almost 15 years. Kaplan-Meier survival analysis suggests that men have higher survival than women. Age of the patient at the time of implant was not a statistically significant factor for re-implantation ( $p$ value $=0.21)$ but sex was $(p$ value $=0.02)$. Women had 2.25 times more risk of being re-implanted than men.
\end{abstract}

Conclusion: In our case series, the failure rate, as a result of aseptic loosening, was $7.4 \%(n=51)$.

More than half the failure cases (56\%) required re-implantation. Over one-third (37\%) of the primary arthrosis cases, were found to be FAI, lessening the frequency of diagnosis of the former. In our clinic, the threaded cup seems to be indispensable in hip revision surgery and in treating FAI.

Keywords: Hip; FAI; Conclusion

Acknowledgements

This article does not contain any studies with human participants or animals performed by any of the authors.

\section{Introduction}

In our hospital, the most common indication for hip revision surgery is post dysplastic arthrosis, followed by femoroacetabular impingement (FAI), early progressive and deformative arthrosis of the hip joint and protrusio acetabuli, with cases of achondroplasia or deformative arthrosis in hereditary multiple exostoses being rarer but not uncommon. The cases of post dysplastic arthrosis represent developmental dysplasia of the hip (DDH), epiphyseal dysplasia, spondylo-epiphyseal dysplasia, and proximal femoral focal deficiency (PFFD) or neurogenic luxation of the hip. In our experience, a small portion of the patients requiring hip revision surgery have shown necrosis of the femoral head, manifesting from chondrolysis of the hip joint, from coxitis (including tuberculosis coxitis), from radiation therapy and from hemophilia. In addition, we noted over time, that a small portion of rheumatic patients were treated with the use of threaded cups.

In our experience, we have also noted that a portion of the patients who were treated with threaded cups experienced complications. The complication of posttraumatic arthrosis has not been uncommon, the conditions of which involve slipped capital femoral epiphyisis, acetabular fractures either treated conservatively or operatively, pseudoarthrosis after femoral neck fractures or fusion of the hip joint. Cancer patients have represented a distinctive group for postsurgical complications (including need for re-implantations). The types of cancers related to the original 
hip revision surgeries have been benign acetabular cysts, Hodgkin's lymphoma, Ewing's sarcoma, and giant cell tumor or desmoplastic fibroma in the supra-acetabular region. In our hospital, the ratio of primo-implantation to total implantations has been relatively low and that of re-implantations has been nearly one-third, making threaded cups irreplaceable in hip revision surgery at our institution.

In order to share the knowledge from our collective experience with hip revision surgery using threaded cups for diverse cases, we performed a systematic evaluation of the overall complications our patients have experienced from hip revision over a 10-year period (2002-2012).

\section{Materials and Methods}

In the 10-year period of 2002-2012, a total of 565 patients underwent hip revision surgery with threaded cups. Of those, 504 patients had complete data and were selected for analysis (189 men and 315 women). The total study population included 49 patients with re-implantation and 455 patients that were censored. For the re-implanted patients, the mean time to re-implantation was 6.3 years and the mean age at the time of implant was 54.7 years. For the censored patients, the mean time to censoring was 5.5 years and the mean age at implant was 55.7 years (Table 1). Among the censored patients, 77 died and 378 did not experience an event necessitating re-implantation.

Table 1: Descriptive statistics of time to event and age of the patient at the time of implant for the study population. Data are presented in years.

\begin{tabular}{|c|c|c|c|c|}
\hline & \multicolumn{2}{|c|}{ Re-implanted patients } & \multicolumn{2}{c|}{ Censored patients } \\
\hline & Time to reimpl. & Age at implant & Time to censoring & Age at implant \\
\hline Mean & 6.32 & 54.68 & 5.46 & 12.34 \\
\hline Stand. dev. & 4.05 & 14.30 & 4.03 & 23.18 \\
\hline Minimum & 0.02 & 14.42 & 0.01 & 51.07 \\
\hline 1. quartile & 3.11 & 47.59 & 2.18 & 58.37 \\
\hline Median & 6.25 & 56.35 & 5.62 & 63.33 \\
\hline 3. quartile & 9.44 & 64.20 & 7.62 & 79.13 \\
\hline Maximum & 14.80 & 86.11 & 15.06 & \\
\hline
\end{tabular}

Among the total study population, $16.8 \%$ had indication for primary arthrosis. In general, those patients were relatively younger and with early progressive and deformative arthrosis of the hip joint, hip arthrosis with protrusion, and FAI. We also recorded the numbers of achondroplasia patients or deformative arthrosis in hereditary multiple exostoses. When we selected 100 patients from among the group of primary arthroses cases, $37 \%$ were found to be FAI, indicating that FAI is secondary arthrosis in origin.

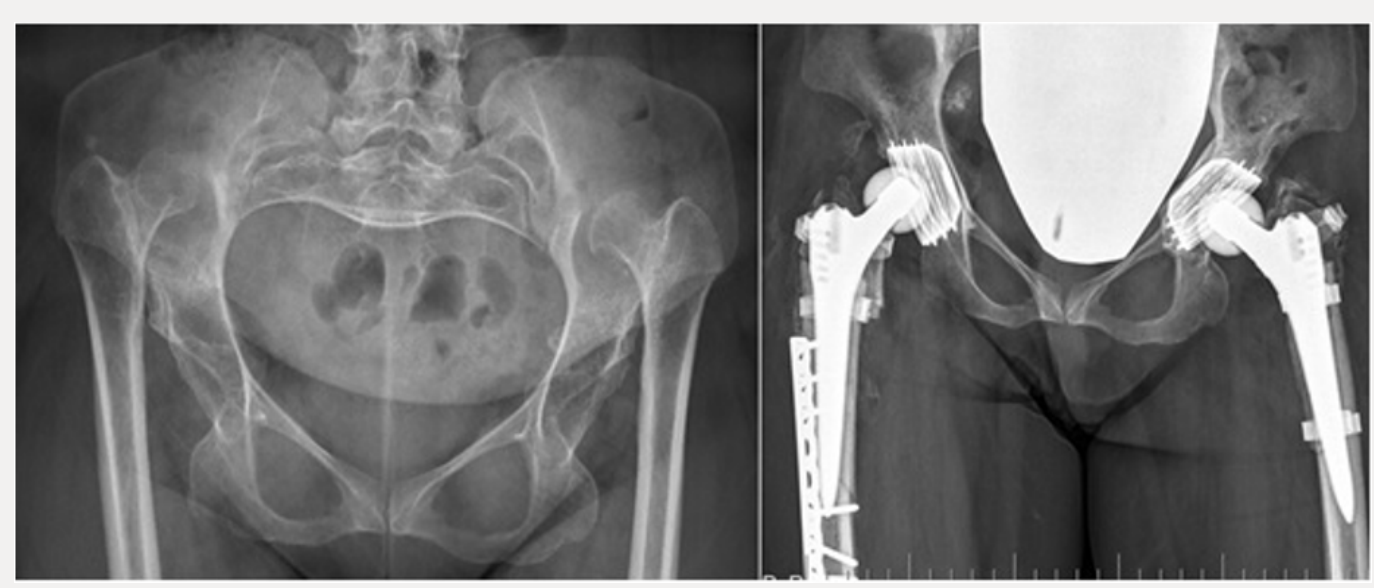

Figure 1: Representative case of high iliac luxation - Hartofilakidis Type III, Crowe Type IV, Eftekhar Type D hip dysplasia, solved with bilateral endoprosthesis (THR) and having visible difficulties with stem implantations.

Patients with post dysplastic arthrosis were the largest group among the hip revision surgery cases (60.3\%). These cases included DDH, epiphyseal dysplasia, spondylo-epiphyseal dysplasia, and PFFD or neurogenic luxation of the hip representing nearly every type of difficult anatomical acetabulum for restoration of true acetabular region or for re-lengthening of the lower extremities (Figure 1).
Among the total study population, $10.4 \%$ had indication of necrosis of the femoral head. These cases consisted of such conditions as chondrolysis of the hip joint, following coxitis (including the stages after tuberculosis coxitis), or necrosis resulting from radiation therapy or in relation to hemophilia (Figure 2). 


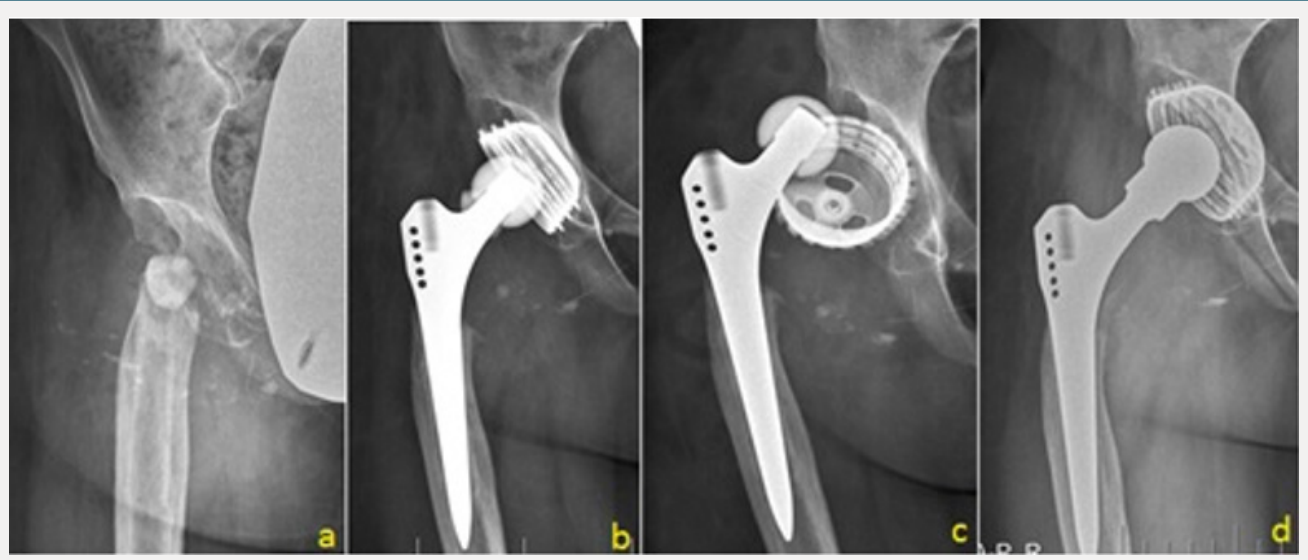

Figure 2: A patient requiring hip revision surgery following septic coxitis with necrosis of the head of the femur. The patient underwent THR. (a) 3-months post-operative. (b) 6-months post-operative. (c) Aseptic loosening of the cup detected at 9 months follow-up. (d) Re-implantation with larger cup.

Rheumatic patients in our study population had received longterm corticoid therapy; among these patients, osteoporosis and necrosis of the femoral head is common. The threaded cup was used in $3.2 \%$ of this study subgroup. The rheumatic patient group also included several cases of ankylosing spondylitis (also known as Bechterev's disease) and juvenile rheumatoid arthritis. Regarding the difficult anatomical acetabulum of these cases, $7.7 \%$ of the patients implanted with threaded cups experienced posttraumatic arthrosis, the conditions of which involved slipped capital femoral epiphyisis, acetabular fractures either treated conservatively or after osteosynthesis, pseudoarthrosis after femoral neck fractures or conditions after posttraumatic arthrodesis of the hip joint.

Among the total study population, rare indication was oncological status (1.6\%). Such cases consisted of benign acetabular cysts, Hodgkin's lymphoma, Ewing's sarcoma, giant cell tumor or desmoplastic fibroma in the supraacetabular region. When we compare threaded THR primo-implantations to total number of primo-implantations at our institution in the 10-year study period, there was an average $13.1 \%$ of primo-implantations. The same comparison for the re-implantation group showed that we used the threaded THR $27.2 \%$ on average, making this treatment modality indispensable in hip revision surgery at our institution.

\section{Results}

In the study presented herein, we focused on cup failures, as the cup has a special threaded design which is a major issue of criticism in regard to implant-related complications. Aseptic loosening was analyzed as it represented the largest amount of complications, being present in $7.4 \%(51 / 689)$ of the total cases studied.

Three patients presented breakage of the metal shell, and in all the breakage was a result of loosening of the bony coverage in the supero-lateral part of the cup. An important aspect of this data is that all breakages occurred among the older types of cups that had been produced before 1995 (i.e. the Zweymueller cup, Smith and Nephew). The design of the cage at that time included 4 notches for the loading device. This type of breakage can be considered as a failure itself, as already found in resistance analyses [1-6] wherein deterioration has been demonstrated to occur over time. There are significant higher stress risers in cups of the older designs for the loader; the modern design, i.e. with 8 notches, overcomes these disadvantages and has a much lower risk of breakage (Figure 3).

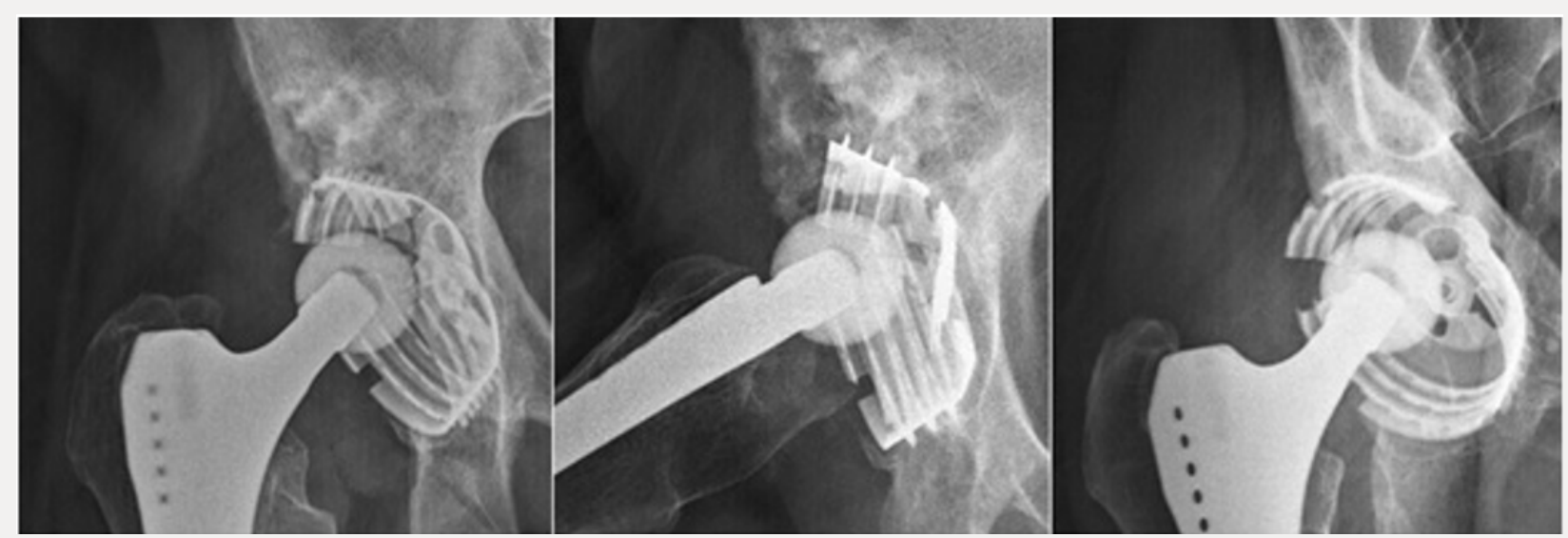

Figure 3: Representative case of cup breakage due to loss of bone in the supero-lateral part of the acetabulum affected with metastatic process. 
We noticed aseptic loosening of the cup in 48 cases, of which 18 cups loosened after primo-implantation and 29 loosened after re-implantation, giving a failure rate of over one-half (56\%). This high percentage is largely due to the area of implantation being technically difficult, which necessitated the use of a different device (i.e. the threaded cup); the rest represented failure of the cups after acetabular fractures ( 2 cases) and after metastatic affections surrounding the acetabulum ( 2 cases). Dislocations of the THR occurred in 4.4\% (30 cases) of the study population, and 15 of the cups required operative intervention. In 5 cases, open reduction was enough; in 9 cases we re-implanted the inlay or head with one of a bigger size, and in 6 cases we performed re-implantation of the stem or cup to correct the position of the implant.

There were infectious complications in 3\% (21 cases) of the study population. In 17 of those cases, a revision procedure was required, including 5 revisions to address an early superficial infection and 2 revisions for deep infection. Proper drainage with retained implant was achieved in all cases. A total of 13 patients remain in follow-up to date, including 3 patients with chronical fistulation, 8 patients who underwent two-stage re-implantation of the THR, and 2 patients who underwent implant removal and had no further re-implantation.

There was slow migration of the implant into the pelvis or up into the iliac bone without obvious loosening in $2.3 \%$. This migration is likely explained by loosening of the circumferential grip of the cortical bone, when the anterior and/or posterior acetabular wall is weakened. In such a condition of cortical weakening, the underlying cancellous bone does not have enough mechanical strength and thus the cup migrates (Figure 4).

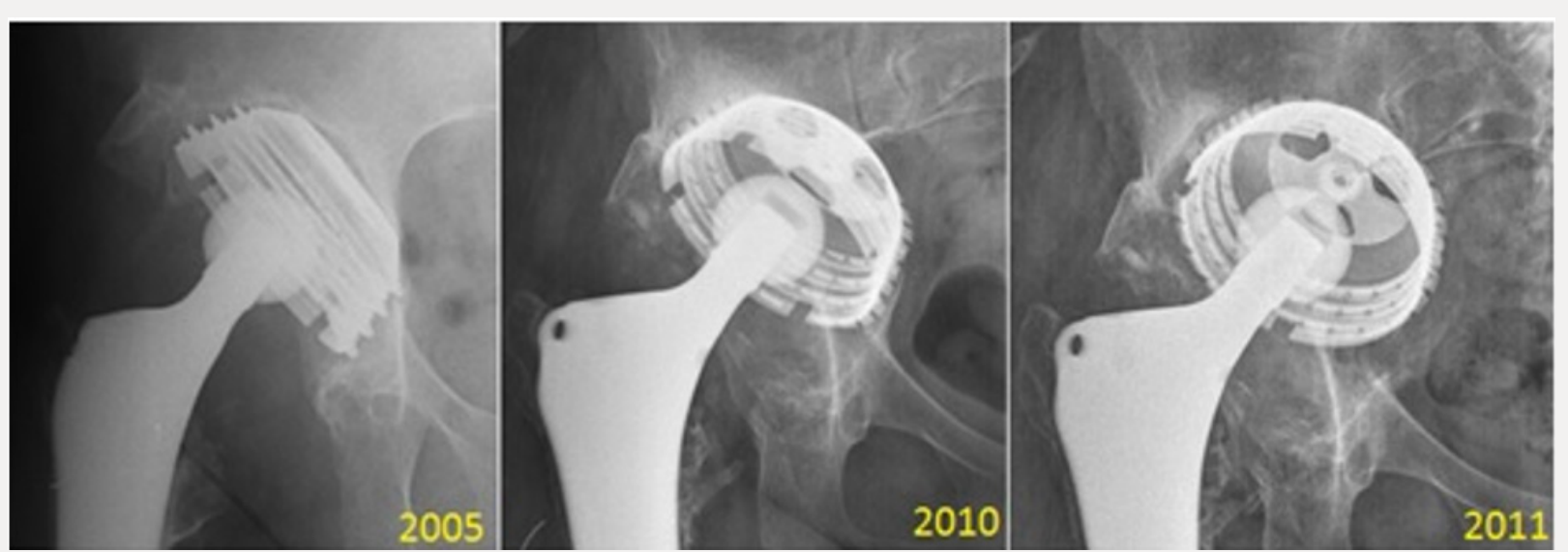

Figure 4: Representative case of slow migration up into iliac bone, with loosening at 8 years after the primary implantation.

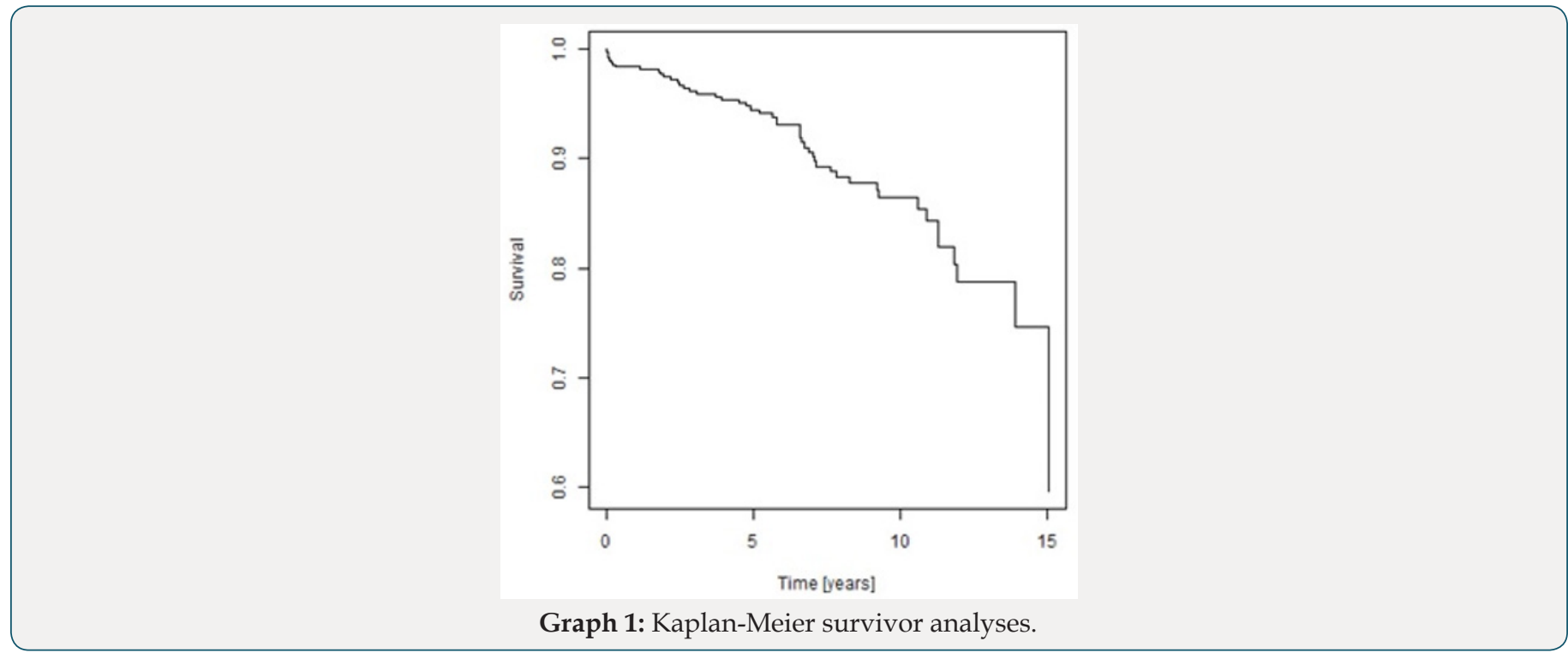

Considering the threaded cup concept, we have observed stress shield effect often. Since the stress shield exerts its effect in cup migration, focused follow-up is necessary to obtain meaningful findings. The advantage of a threaded cup is that there is no need of full coverage. In particular, $70 \%$ of coverage is sufficient, without need of augmentation [1]. In addition, arthritis with protrusion (FAI pincer type) is one of the indications for the threaded cup since those cases do not need support of the acetabular floor. Implantrelated complications are to be considered as neuro-vascular complications. Among our study population, there was a total 
number of 10 nerve palsies (1.5\%), 4 of which had to be operated; two of those patients underwent sural graft transplantation due to femoral nerve cutoff, [2] and the other two underwent the same procedure for static nerve palsy, with one requiring simultaneous anterior tibial tendon transfer. In one case, we released the lateral cutaneous nerve (Graph 1). The Kaplan-Meier survical curve suggests that men have higher survival than women do (Graph 2).

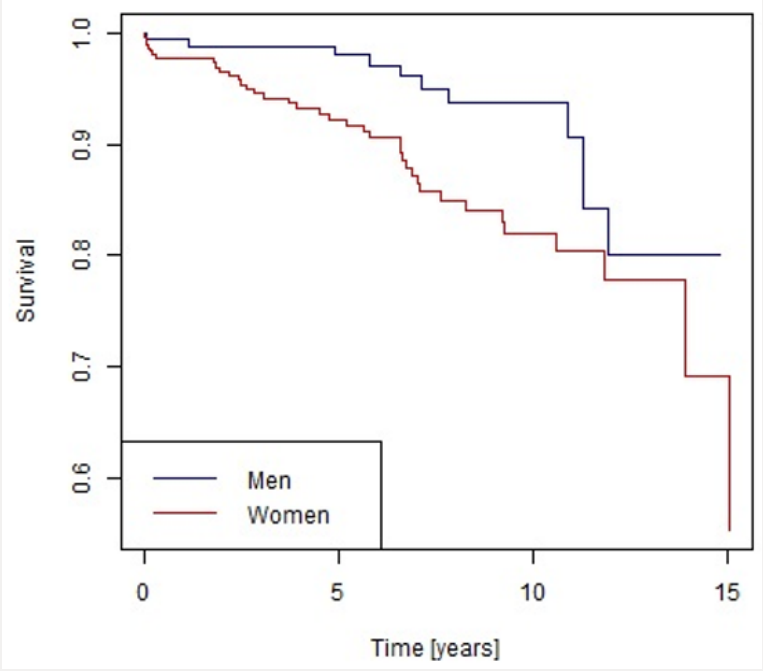

Graph 2: Kaplan-Meier survivor curve for men and women, showing the association of time to event to sex.

Age of the patient at the time of implantation was not statistically significant $(\mathrm{p}$ value $=0.21$ ). Sex was a statistically significant factor ( $\mathrm{p}$ value 0.02 ). Women had 2.25 times more risk of being re-implanted than men (Table 2).

Table 2: Cox model of proportional hazard for patient sex, showing dependence of time to event with age at the implant and sex.

\begin{tabular}{|c|c|c|c|c|}
\hline & Hazard & Stand. error & Confidence interval & $\boldsymbol{p}$ value \\
\hline Women & 2.247 & 1.410 & $(1.145,4.407)$ & 0.019 \\
\hline
\end{tabular}

\section{Conclusion}

Aseptic loosening reportedly occurs in around $6.5 \%$ of implanted patients at 4 years following placement [3]. In one study the radiological sign of instability was found in $21 \%$ of patients (n $=130$ ) at a mean follow-up of 3.9 years [4]. In another study [5], 17 cups (accounting for $25 \%$ of the cases assessed) were revised at an average of 5.2 years, with another 9 additional cases awaiting revision. Brujin et al. [2] reported migration for $25 \%$ of threaded cups in a follow-up of 4.5 years, and a $6 \%$ revision rate. It has been suggested that high and continuous stress near the threads may not allow bony in-growth [6] and may produce ischemia and necrosis [7] that require surgical revision in 14 months following the implantation surgery [8].

\section{Discussion}

Overall, $7.4 \%$ of our patients experienced loosening of the threaded cups and 3\% experienced infectious loosening, which agrees with the literature $[9,10]$. In general, loosening of threaded cups does not give a good clinical and/or radiological outcome over the long term. X-ray appearance deteriorates with time, without evidence of an initial clinical correlation. The appearance of relative lines does not necessarily equate to the occurrence of loosening, but such a finding should be monitored carefully thereafter. Detection of osteolysis, however, indicates that loosening will occur [11]. A study by Harris et al from 1986 indicated that 79\% of coxartosis cases originated from the cam impingement, with the rest by rheumatoid arthritis (21\%) and hemochromatosis (10\%).

In our series, when we compared 100 patients admitted for the THR procedure, $37 \%$ showed FAI signs, with the average age of implantation being 58 years old. There were $23 \%$ post-DDH cases, $11 \%$ idiopathic avascular necrosis, $17 \%$ post-trauma cases, and $12 \%$ others; thus, the diagnosis of primary arthrosis is much less common. FAI both types are finally resolved by a THR and indicated much earlier than primary arthrosis. As changing in orientation of the acetabuli we use these threaded cups, [12] more in pincer type of the FAI, where orientation is essential. We don't necessarily use these cups, though in special cases we have to prefer primary stability to have good long-term results. The failure of the threaded acetabular component is due to difficulties of anatomy of the acetabulum, as observed in the cases in which we used this component.

Even though we decided to use this special design of threaded cup, despite its higher rate of complications, we were able to resolve one-third of our re-implantation cases and to overcome the difficulties of the acetabulum in FAI $[13,14]$. Kaplan-Meier survival curve analyses identified threaded cups as a good option (with survival of 10 years); the Cox proportional curve indicated higher risk for re-implant for women. Even though we investigated THR as a last option for patients with FAI, pelvic transfers or 
any cervicocapital remodeling may also represent last options. Ultimately, this study did not show the threaded cup to be ideal for complicated acetabulum, though in our clinic it is ideal for this diagnosis.

\section{Informed Consent Disclaimer}

This article does not contain any studies actively involving human participants or animals.

\section{References}

1. Apel DM, Smith DG, Schwarz CM, Paprosky WG (1989) Threaded cup acetabuloplasty. Early clinical experience. Clin Orthop 241: 183-189.

2. Bruijn JD, Seelen RM, Feenstra RM, Hansen BE, Bernoski FP (1995) Failure of the Mecring screw-ring acetabular component in total hip artroplasty. A three to seven-year follow-up study. J Bone Joint Surg 77: 760-766

3. Engh CA, Griffin WL, Marxc C (1990) Cementless acetabular components. J Bone Joint Surg 72(1): 53-59.

4. Fox GM, McBeath AA, Heiner JP (1994) Hip replacement with a threaded acetabular cup. A follow-up study. J Bone Joint Surg 76(2): 195-201.

5. Herberds P, Malchau H, Romanus B, Wiklund LM (1986) Lord's cementless total hip replacement in young patients: 2-7 years result. Orthop Trans 10: 442-446.

6. Shaw JA, Bailey JH, Bruno A, Greer RB III (1990) Threaded acetabular components for primary and revision total hip arthroplasty. J Arthoplasty 5(3): 201-215.
7. Huiskes R (1987) Finite element analysis of acetabular reconstruction. Noncemented threded cups. Acta Orthop Scand 58: 620-625.

8. Kody MH, Kabo JM, Markolf KL, Dorey FJ, Amstutz HC (1990) Strenght of intial mechanical fixation of screw ring acetaular components. Clin Orhop 257: 146-153.

9. Maloney WJ, Smith RL (1995) Periprostetic osteolysis in total hip arthoplasty. The role of particulate wear debris. J Bone Joint Surg 77(9): 1448-1461.

10. Schmalzried TP, Jasty M, Harris WH (1992) Perisprosthetic bone loss in total hip arthoplasty. Polyethylene wear debris and the concept of the effective joint space. J Bone Joint Surg 74(6): 849-863.

11. Tallroth K, Slätis P, Ylinen P, Paavolainen P, Paavolainen T (1993) Loosening of threaded acetabular components. Radiographic manifestations. J Arthoplasty 8(6): 581-584

12. Yiannakopoulos CK,Chougle A, Eskelinen A, Hodgkinson JP, Hartofilakidis G (2008) Inter-and intraobserver variability of the Crowe and Hartofilakidis classification systems for congenital hip disease in adults. J Bone Jt Surg 90(5): 579-583.

13. Hartofilakidis G, Stamos K, Karachalios T, Ioannidis TT, Zacharakis N (1996) Congenital hip disease in adults. Clasification of acetabular deficiencies and operative treatment with acetabuloplasty combined with total hip arthroplasty. J Bone Jt Surg 78(5): 683-692.

14. Hartofilakidis G,Stamos K,Karachalios T (1998) Treatment of High Dislocation of the Hip in Adults with Total Hip Arthroplasty. JBone Jt Surg 80(4): 510-517.

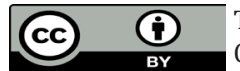

This work is licensed under Creative Commons Attribution 4.0 License

To Submit Your Article Click Here:

Submit Article

DOI: 10.32474/LOJMS.2018.02.000132

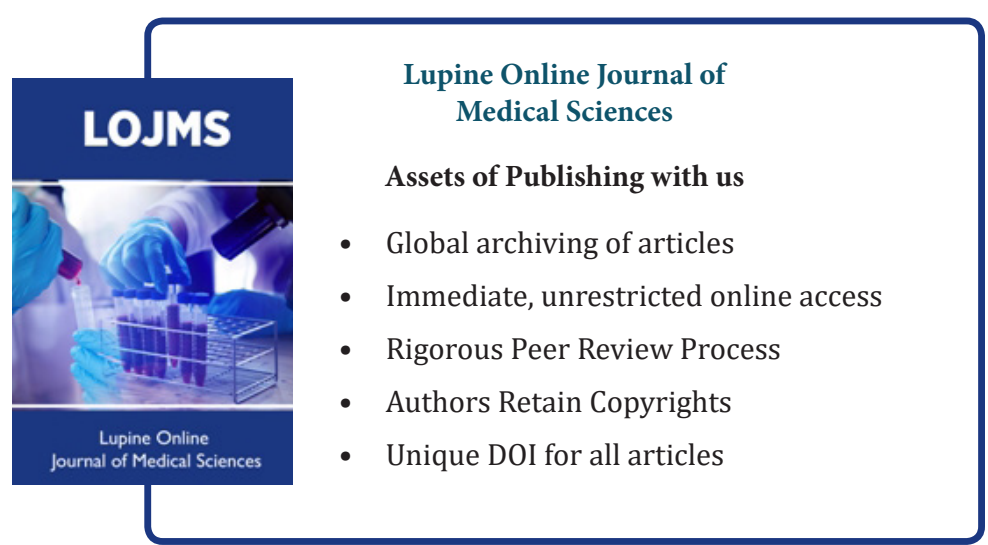

